\title{
A novel fiber-2-edited live attenuated vaccine candidate against the highly pathogenic serotype 4 fowl adenovirus
}

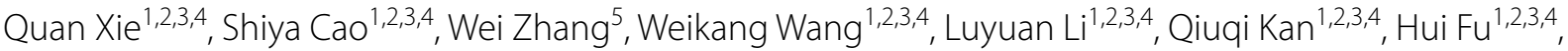 \\ Tuoyu Geng ${ }^{6}$, Tuofan Li1,2,3,4, Zhimin Wan ${ }^{1,2,3,4}$, Wei Gao ${ }^{1,2,3,4}$, Hongxia Shao 1,2,3,4 , Aijian Qin 1,2,3,4 \\ and Jianqiang $Y e^{1,2,3,4^{*}}$
}

\begin{abstract}
Recently, the outbreaks of hydropericardium-hepatitis syndrome (HHS) caused by the highly pathogenic fowl adenovirus serotype 4 (FAdV-4) have resulted in huge economic losses to the poultry industry globally. Although several inactivated or subunit vaccines have been developed against FAdV-4, live-attenuated vaccines for FAdV-4 are rarely reported. In this study, a recombinant virus FA4-EGFP expressing EGFP-Fiber-2 fusion protein was generated by the CRISPR/Cas9 technique. Although FA4-EGFP shows slightly lower replication ability than the wild type (WT) FAdV4, FA4-EGFP was significantly attenuated in vivo compared with the WT FAdV-4. Chickens infected with FA4-EGFP did not show any clinical signs, and all survived to 14 day post-infection (dpi), whereas those infected with FAdV-4 showed severe clinical signs with HHS and all died at 4 dpi. Besides, the inoculation of FA4-EGFP in chickens provided efficient protection against lethal challenge with FAdV-4. Compared with an inactivated vaccine, FA4-EGFP induced neutralizing antibodies with higher titers earlier. All these data not only provide a live-attenuated vaccine candidate against the highly pathogenic FAdV-4 but also give a potential insertion site for developing FAdV-4-based vaccine vectors for delivering foreign antigens.
\end{abstract}

Keywords: FAdV-4, CRISPR/Cas9, Recombinant virus, Attenuation, Vaccine candidate

\section{Introduction}

Fowl adenoviruses (FAdV) are non-enveloped viruses with a double-stranded DNA genome, belonging to Adenoviridea and genus Aviadenovirus [1]. Based on the profile of restriction enzyme digestion and the sera cross-neutralization assay, FAdV has been clustered into 5 species (FAdV-A $\sim$ E) with 12 serotypes (FAdV-1 to $8 \mathrm{a}$ and $8 \mathrm{~b}$ to 11 ) [2]. The infection of FAdV mainly causes clinical symptoms, including inclusion body hepatitis

\footnotetext{
*Correspondence: jqye@yzu.edu.cn

${ }^{1}$ Key Laboratory of Jiangsu Preventive Veterinary Medicine, Key Laboratory for Avian Preventive Medicine, Ministry of Education, College of Veterinary Medicine, Yangzhou University, Yangzhou 225009, Jiangsu, China

Full list of author information is available at the end of the article
}

(IBH), hepatitis-hydropericardium syndrome (HHS), and gizzard erosion and ulceration (GEU) [3]. Among the 12 serotypes of FAdV, FAdV-4 is the main causative agent for HHS in chickens [4-6]. It is noteworthy that the recent outbreaks of HHS caused by the highly pathogenic FAdV-4 have resulted in huge economic losses to the poultry industry worldwide [7-10]. Many inactivated or subunit vaccines have been developed to control HHS [3]. However, the live-attenuated vaccine against FAdV-4 has rarely been reported $[11,12]$.

Unlike other serotypes, serotypes FAdV-1, FAdV-4, and FAdV-10 have two fiber genes (fiber-1 and fiber-2) [13]. Recently, our group and Wang's group found that Fiber1 , but not Fiber-2, directly triggered the viral infection of FAdV-4 via its shaft and knob domains [14, 15]. However,

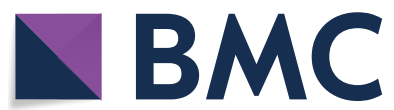

(c) The Author(s) 2021. This article is licensed under a Creative Commons Attribution 4.0 International License, which permits use, sharing, adaptation, distribution and reproduction in any medium or format, as long as you give appropriate credit to the original author(s) and the source, provide a link to the Creative Commons licence, and indicate if changes were made. The images or other third party material in this article are included in the article's Creative Commons licence, unless indicated otherwise in a credit line to the material. If material is not included in the article's Creative Commons licence and your intended use is not permitted by statutory regulation or exceeds the permitted use, you will need to obtain permission directly from the copyright holder. To view a copy of this licence, visit http://creativeco mmons.org/licenses/by/4.0/. The Creative Commons Public Domain Dedication waiver (http://creativecommons.org/publicdomain/ zero/1.0/) applies to the data made available in this article, unless otherwise stated in a credit line to the data. 
Zhang et al. reported that Fiber-2, but not Fiber-1, was identified as one of the major virulent determiners for the highly pathogenic FAdV-4 endemic in China [16]. These findings indicate that Fiber-1, but not Fiber-2, is required for the infection of FAdV-4, whereas Fiber-2, but not Fiber-1, plays vital roles in the pathogenicity of FAdV-4. Therefore, Fiber- 2 might be an efficient target for developing live-attenuated vaccine candidates against FAdV-4. Here, we used the CRISPR/Cas9 approach to target the fiber-2 gene of the wild type (WT) FAdV-4 to generate a recombinant virus FA4-EGFP expressing EGFP-Fiber-2 fusion protein. In vitro and in vivo studies demonstrated that FA4-EGFP is highly attenuated compared with the WT FAdV-4 and could provide efficient protection against lethal challenge with WT FAdV-4.

\section{Materials and methods}

\section{Cells, viruses, and antibodies}

The FAdV-4 strain SD was isolated and stored in our laboratory and propagated in leghorn male hepatoma (LMH) cells. LMH cells were purchased from ATCC and cultured in Dulbecco Modified Eagle Medium/F12 (Gibco, NY, USA) supplemented with $10 \%$ fetal bovine serum (Lonsera, Shanghai, China) in a $5 \% \mathrm{CO}_{2}$ incubator at $37{ }^{\circ} \mathrm{C}$. Monoclonal antibody (mAb) $3 \mathrm{~B} 5$ against Fiber1 , and $\mathrm{mAb} 3 \mathrm{C} 2$ against Fiber-2 were generated in our laboratory, and $\mathrm{mAb} 3 \mathrm{C} 2$ against Fiber-2 were generated in our laboratory, and Professor Hongjun Chen kindly provided mAb 1C9 against Fiber-2.

\section{Construction of sgRNA and donor plasmids}

The sgRNA targeting fiber-2 of the FAdV-4 genome was designed using the CRISPR guide RNA designing website [17] and cloned into the CRISPR/Cas9 vector lentiCRISPR v2. The sequences of the sgRNA are listed in Table 1. The donor plasmid containing the EGFP sequence at the $\mathrm{N}$ terminus of fiber-2 was constructed by several rounds of overlapping PCR. The homologous arm (HA) designed at both ends was $1000 \mathrm{bp}$ in length, respectively. The template was assembled as the HALEGFP-F2-HAR and finally cloned into the pMD19 simple vector. The primers used for constructing donor plasmid are provided in Table 2 .

Table 1 List of primers used for sgRNA cloning

\begin{tabular}{ll}
\hline sgRNA1 & Sequences of primers $\left(\mathbf{5}^{\prime} \mathbf{-} \mathbf{3}^{\prime}\right)$ \\
sgRNA2 & F: CACCGGGTTTATCCTTTCGATTACG \\
& F: AACACCGTAATCGAAAGGATAAACCC \\
& R: AAACCTGGACAGCTGTAGAGCACGC \\
\hline
\end{tabular}

Table 2 PCR primers for constructing donor plasmid and detecting the recombinant virus

\begin{tabular}{ll}
\hline PCR products & Sequences of primers $\left(\mathbf{5}^{\prime} \mathbf{-} \mathbf{3}^{\prime} \mathbf{)}\right.$ \\
\hline HAL & F: GGTGACCTACTGACCCTCAACACC \\
& R: CAGCTCCTCGCCCTTGCTCACCATTGTTCCCGTTGG \\
GGGA & F: TCCCCCAACGGGAACAATGGTGAGCAAGGGCGAGGA \\
EGFP & GCTG \\
& R: CTTCTTTTAGGGGCCCGGAGCTTGTACAGCTCGTCCATG \\
fiber-2+HAR & F: CATGGACGAGCTGTACAAGCTCCGGGCCCTAAAAG \\
& AAG \\
& R: CTACTTTACCTGCATTTCGTCAG \\
Partial fiber-2 & F: CTCCAACTGGTTGACCAGAACG \\
& R: GTCAAGCTGGGATGCTCTCACCATGC
\end{tabular}

\section{Generation of the recombinant FAdV-4-EGFP}

LMH cells were transfected with the sgRNA targeting both ends of the fiber- 2 gene with $2 \mu \mathrm{g}$ of each sgRNA $24 \mathrm{~h}$ post-transfection (hpt), the LMH cells were infected with FAdV-4 at $0.1 \mathrm{MOI}$ and then were transfected with $4 \mu \mathrm{g}$ of the donor plasmid. The infected LMH cells were observed through a fluorescence microscope 24 hours post-infection (hpi). The recombinant virus, designated as FA4-EGFP, was purified by limiting the dilution assay and virus plaque assay. The purified virus was further identified by Western blot, PCR, and sequencing.

\section{Growth curve of the FAdV-4-EGFP in LMH cells}

To determine the replication capacity of the recombinant virus FA4-EGFP, LMH cells seeded in a 6-well plate (about $1.2 \times 10^{6}$ cells per well) were infected with WT FAdV-4 and the recombinant virus FA4-EGFP at 0.1 MOI, respectively, and then the viruses were harvested at $24,48,72,96$ and $120 \mathrm{hpi}$, and stored at $-80{ }^{\circ} \mathrm{C}$ until use. The $\mathrm{TCID}_{50}$ of the harvested viruses were determined in 96-well plates by serial dilution from $10^{-1}$ to $10^{-8}$, and detected at 72 hpi by IFA and calculated by the ReedMuench method.

\section{Western blot assay}

The LMH cells infected with FAdV were collected and lysed in lysis buffer (CST, MA, USA) with PMSF (Beyotime, Shanghai, China), protease and phosphatase inhibitors (CST, MA, USA). The lysates were boiled in the loading buffer and were then subjected to $10 \%$ SDSPAGE and transferred to nitrocellulose (NC) membranes (GE Healthcare Life sciences, Freiburg, Germany). After blocking with 5\% skimmed milk in PBST for $1 \mathrm{~h}$ at room temperature (RT), the membranes were reacted with the corresponding antibodies at $4{ }^{\circ} \mathrm{C}$ overnight. After being 
washed with PBST three times, the membrane was incubated with HRP-labelled secondary antibodies for $1 \mathrm{~h}$ at RT. After another three washes, the membranes were developed with chemiluminescent reagents and imaged with an automatic imaging system (Tanon 5200).

\section{Indirect immunofluorescent assay}

The LMH cells infected with viruses were fixed with prechilled acetone: ethanol $(3: 2 \mathrm{v} / \mathrm{v})$ mixture for $5 \mathrm{~min}$ at RT and washed with PBS. The cells were then incubated with the diluted mAb 3B5 against Fiber- 1 for $45 \mathrm{~min}$ at $37{ }^{\circ} \mathrm{C}$. After washing three times with PBS, the cells were incubated with the diluted second antibody (goat anti-mouse IgG-FITC) for another $45 \mathrm{~min}$ at $37^{\circ} \mathrm{C}$. Again, after three washes with PBS, the cells were observed by invert fluorescence microscopy.

\section{Animal experiments}

For the pathogenesis analysis, a total of 120 one-dayold SPF chickens were randomly divided into three groups (40 chickens per group; Positive control group: chickens infected with FAdV-4; Experiment group: chickens infected FA4-EGFP; Negative control group: chickens inoculated with $1 \%$ culture medium). The chickens were housed in different negative-pressure isolators for 14 days and subsequently infected with $10^{6} \mathrm{TCID}_{50}$ of the indicated virus in $200 \mu \mathrm{L}$ of $1 \%$ culture medium intramuscularly, the negative control group was inoculated with the same volume of $1 \%$ culture medium. On 2, 3, 4, 5, $6 \mathrm{dpi}$, the cloacal swabs were collected, and three chickens in each group were euthanized; the liver, the spleen, the kidney was collected for viral titration or histopathology analysis. For the protective analysis, the chickens survived in the experimental group, and the negative control group at $21 \mathrm{dpi}$ were challenged with $10^{6}$ $\mathrm{TCID}_{50}$ of FAdV-4 in $200 \mu \mathrm{L}$ of $1 \%$ culture medium intramuscularly. On 2, 3, 4, 6, 8 days post-challenge (dpc), the cloacal swabs were collected, and three chickens in each group were euthanized; the liver, the spleen, the kidney was collected for viral titration. The clinical symptoms and mortality of the infected or challenged chickens were monitored daily.

To further compare the protective efficacy of FA4EGFP with the inactivated FAdV-4 vaccine candidate, a total of 50 2-week old SPF chickens were randomly divided into 5 groups. Three groups were inoculated intramuscularly with the three doses of FA4-EGFP $\left(10^{6}\right.$ $\mathrm{TCID}_{50}, 10^{5} \mathrm{TCID}_{50}, 10^{4} \mathrm{TCID}_{50}$ ) in $200 \mu \mathrm{L}$ of $1 \%$ culture medium, respectively. The other two groups were inoculated with the inactivated oil-emulsion FAdV-4 containing $5 \times 10^{6} \mathrm{TCID}_{50}$ of viruses and the same emulsion volume intramuscularly, respectively. On 7, 14, $21 \mathrm{dpi}$, the blood was collected for the neutralization test. On 21 dpi, chickens were challenged with $10^{6} \mathrm{TCID}_{50}$ of FAdV-4 in $200 \mu \mathrm{L}$ of $1 \%$ culture medium, and the survival data was recorded. The clinical symptoms and mortality of the challenged chickens were monitored daily.

All animal experiments were performed following the "Guidelines for Experimental Animals" and the protocol (SYXY-19), which was approved by the Animal Care and Use Committee of Yangzhou University (Yangzhou, China). At the end of the experiment, all the chickens were euthanized by $\mathrm{CO}_{2}$.

\section{Neutralization test}

Different chicken sera dilutions were first mixed with 1000 TCID $_{50}$ of FAdV-4 and incubated for $1 \mathrm{~h}$ at $37{ }^{\circ} \mathrm{C}$. The mixtures were then added to the 96 -well plate with $\mathrm{LMH}$ cells and incubated for $2 \mathrm{~h}$ at $37^{\circ} \mathrm{C}$. After washing once, the cells were cultured in F12/DMEM medium with $1 \%$ fetal bovine serum. After being cultured for $96 \mathrm{~h}$, the cells were fixed and subjected to IFA analysis using $\mathrm{mAb}$ 3B5 against Fiber-1 of FAdV-4 as previously described.

\section{Titration of viral titer in organs and cloacal swabs}

The liver, spleen, and kidney collected were homogenized, treated with tenfold penicillin and streptomycin for $1 \mathrm{~h}$, and centrifuged to obtain the supernatant. The cloacal swabs collected from the chickens were placed in $800 \mu \mathrm{L}$ of PBS. After three times of freeze-thaw cycles, the samples were treated the same with organ homogenates. The virus-containing supernatants were then serially diluted and inoculated into LMH cells. The infected LMH cells were fixed and detected by IFA using $3 \mathrm{~B} 5 \mathrm{mAb}$ against Fiber- $14 \mathrm{dpi}$, and the $\mathrm{TCID}_{50}$ of these supernatants was determined by the Reed-Muench method.

\section{Statistical analysis}

All the results are presented as means \pm standard deviation. This study's statistical analysis was performed with a Student test or One-way ANOVA test using GraphPad 5 software. $P$-value of $<0.05$ was considered significant. *, $* *$, and $* *$ indicate $P$-value less than $0.05,0.01$ and 0.001 , respectively.

\section{Results}

Generation of recombinant virus FA4-EGFP expressing EGFP-Fiber-2 protein

To evaluate whether the fiber-2 gene of FAdV-4 could be an efficient target for developing live-attenuated vaccine candidates against FAdV-4 or vaccine vectors for expressing foreign antigens, two sgRNA targeting $\mathrm{N}$ - and C-terminus of fiber-2, respectively, were first designed and cloned into lentiCRISPR v2, and the donor plasmid containing the EGFP sequence at the $\mathrm{N}$ terminus of fiber-2 was constructed by overlap PCR as described 


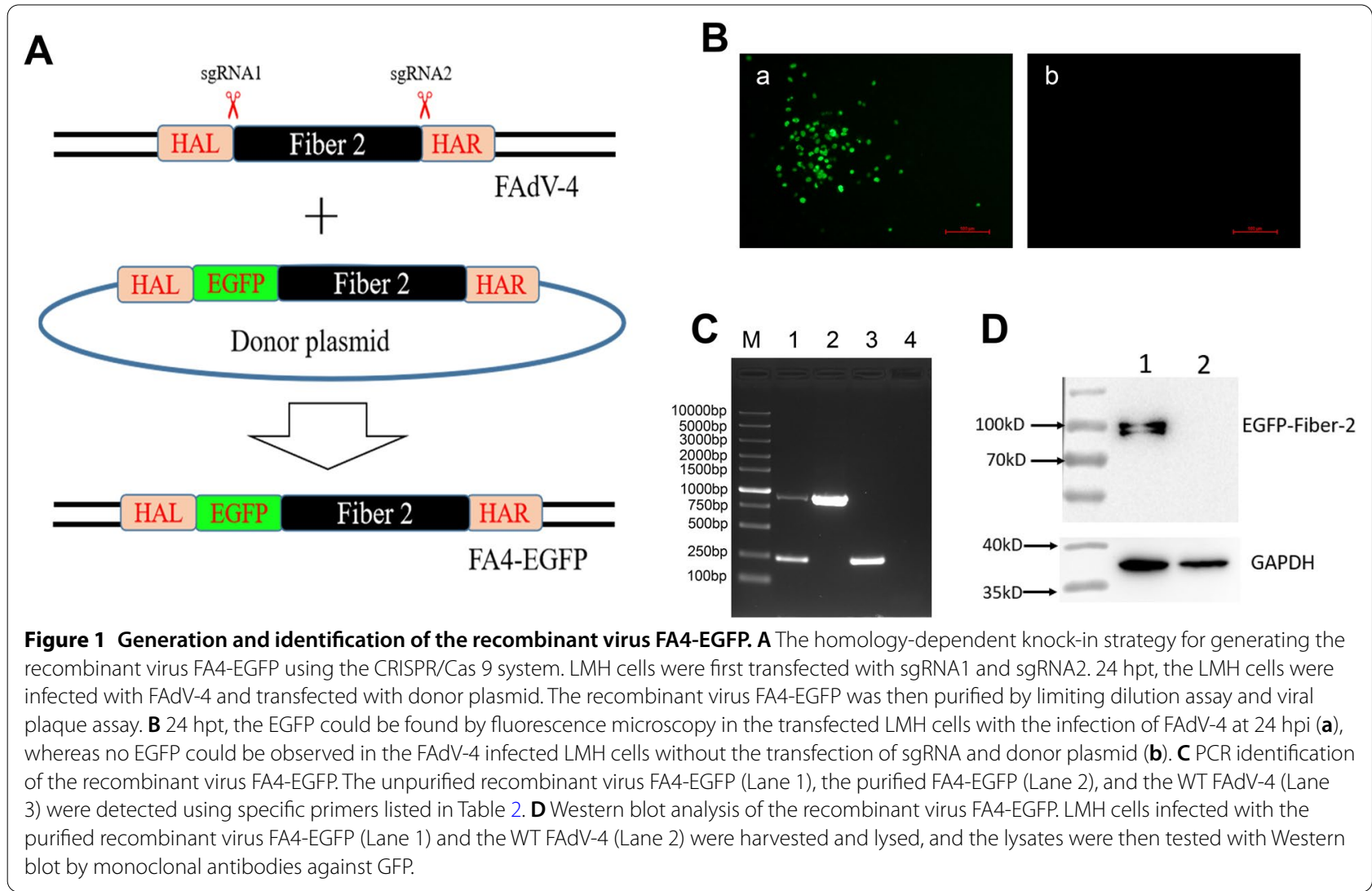

in Figure 1A. A recombinant virus, designated as FA4EGFP expressing EGFP-Fiber-2 fusion protein, was generated through the transfection of the two sgRNA and the donor plasmid and the infection of FAdV-4 in LMH cells. Notably, the virus plagues with EGFP could be found in the transfected LMH cells with the infection of FAdV-4 at $24 \mathrm{hpi}$, whereas no EGFP could be observed in the FAdV-4 infected LMH cells without the transfection of sgRNA and donor plasmid (Figure 1B), indicating the recombinant virus FA4-EGFP was efficiently generated. After serial limit dilution and plague purification, the purified FA4-EGFP was further identified by sequencing, Western blot, and PCR. As shown in Figure 1C, the partial egfp-fiber-2 fusion gene was amplified in the purified FA4-EGFP, but not in FAdV-4. Both partial egfp-fiber-2 fusion gene and partial fiber-2 gene were amplified in the unpurified FA4-EGFP, whereas only partial fiber-2 gene was amplified in FAdV-4 using specific primers as listed in Table 2. In the Western blot, the EGFP-Fiber-2 fusion protein was efficiently detected in the LMH cells infected with the purified FA4-EGFP as described in Figure 1D. The conformed sequence of the recombinant GFPFiber-2 in FA4-EGFP are provided as Additional file 1. All these data demonstrate that a novel recombinant virus FA4-EGFP expressing EGFP-Fiber-2 protein is generated.

\section{FA4-EGFP replicated slower than the WT FAdV-4 in vitro}

To compare the growth kinetics of the recombinant virus FA4-EGFP with the WT FAdV-4, LMH cells were infected with the same dose of FA4-EGFP and FAdV-4, respectively, and then the virus supernatants from the infected LMH cells at different time points were collected and titrated. As described in Figure 2A, although FA4-EGFP could efficiently grow in LMH cells, FA4EGFP replicated slightly slower than WT FAdV-4. Notably, the peak titer of FAdV-4 could reach $10^{8} \mathrm{TCID}_{50} / \mathrm{mL}$ while the FA4-EGFP could only reach $10^{7} \mathrm{TCID}_{50} / \mathrm{mL}$ within 5 days. This $\mathrm{TCID}_{50}$ data for the growth kinetics was also confirmed by Western blot analysis. As shown in Figure $2 \mathrm{~B}$, the Fiber-2 protein with a strong strip in the LMH cells infected with FAdV-4 could be efficiently detected at $48 \mathrm{hpi}$ and $72 \mathrm{hpi}$, whereas the band of the Fiber-2 protein in the LMH cells infected with FA4-EGFP was weak. All these demonstrate that although the replication ability of FA4-EGFP is weaker than that of WT FAdV-4, FA4-EGFP can efficiently replicate in LMH cells with high viral titer. 


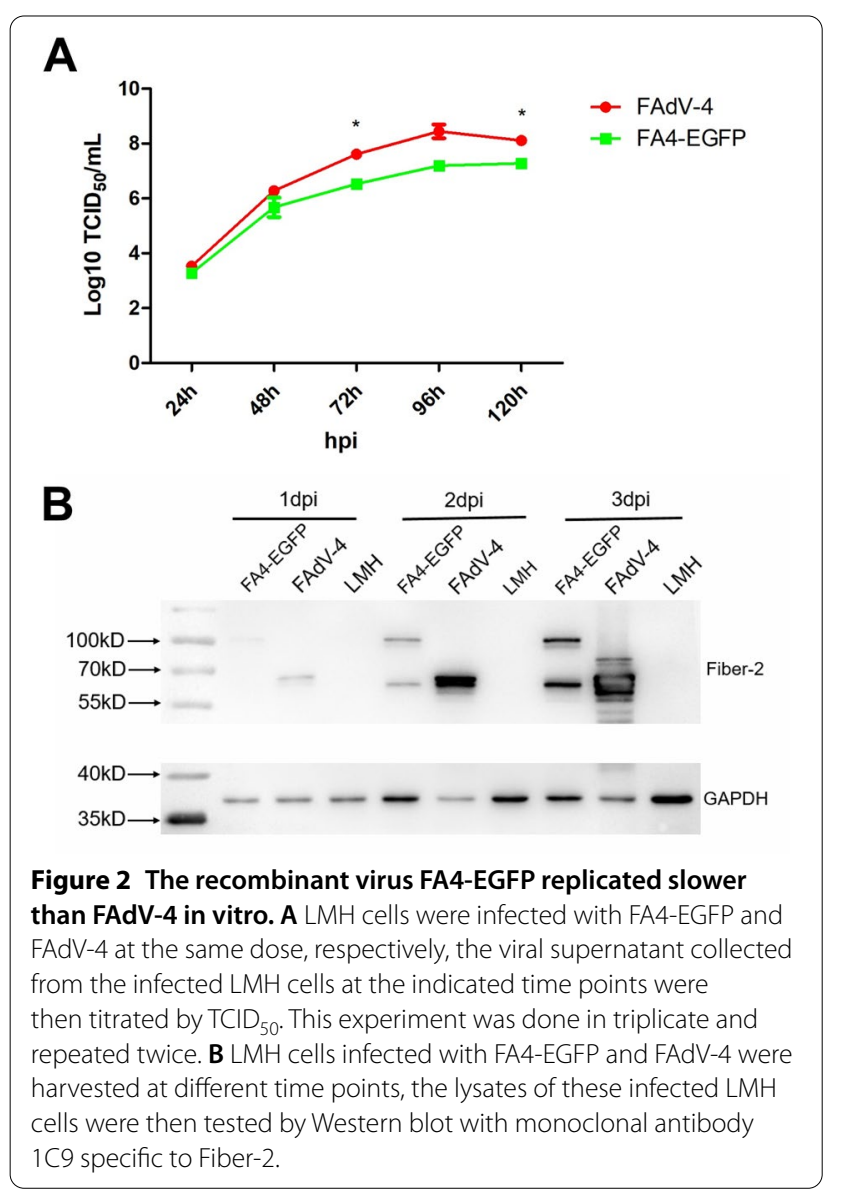

\section{FA4-EGFP was highly attenuated in vivo}

To evaluate the viral replication and pathogenesis of FA4-EGFP in vivo, SPF chickens were infected with FA4-EGFP and wild-type FAdV-4 at the same dose. The infected chickens' clinical symptoms and mortality were monitored daily, and the liver, spleen, kidney, and cloacal swabs were collected for viral titration at the indicated time points. At $2 \mathrm{dpi}$, the chickens infected with FAdV-4 began to show clinical signs characterized by depression, sleepiness and huddling together with ruffled feathers, and all the chickens died within $4 \mathrm{dpi}$ as described in Figure 3A. However, all the chickens infected with FA4-EGFP did not show any clinical signs and death throughout the experiment (Figure 3A). Necropsy analysis demonstrates that the chickens infected with FAdV-4 show HHS, whereas no gross lesion in the heart, liver, spleen, or kidney was observed in the chickens infected with FA4-EGFP (data not shown). The histopathological analysis further demonstrated that the degeneration and necrosis of hepatocytes and the intranuclear inclusion bodies in hepatocytes were observed in the chickens infected with FAdV-4, whereas no obvious histopathological symptoms were found in the chickens infected with FA4-EGFP as shown in Figure 3B. For virus shedding, as shown in Figure $3 \mathrm{C}$, the high viral titer about $10^{4}-10^{5} \mathrm{TCID}_{50} / \mathrm{mL}$ was detected at $2-4 \mathrm{dpi}$ in the cloacal swab samples from chickens infected with FAdV-4, whereas the low viral titer was detected at $2 \mathrm{dpi}$ in the cloacal swab samples of chickens infected with FA4-EGFP. Chickens in the FA4-EGFP group did not

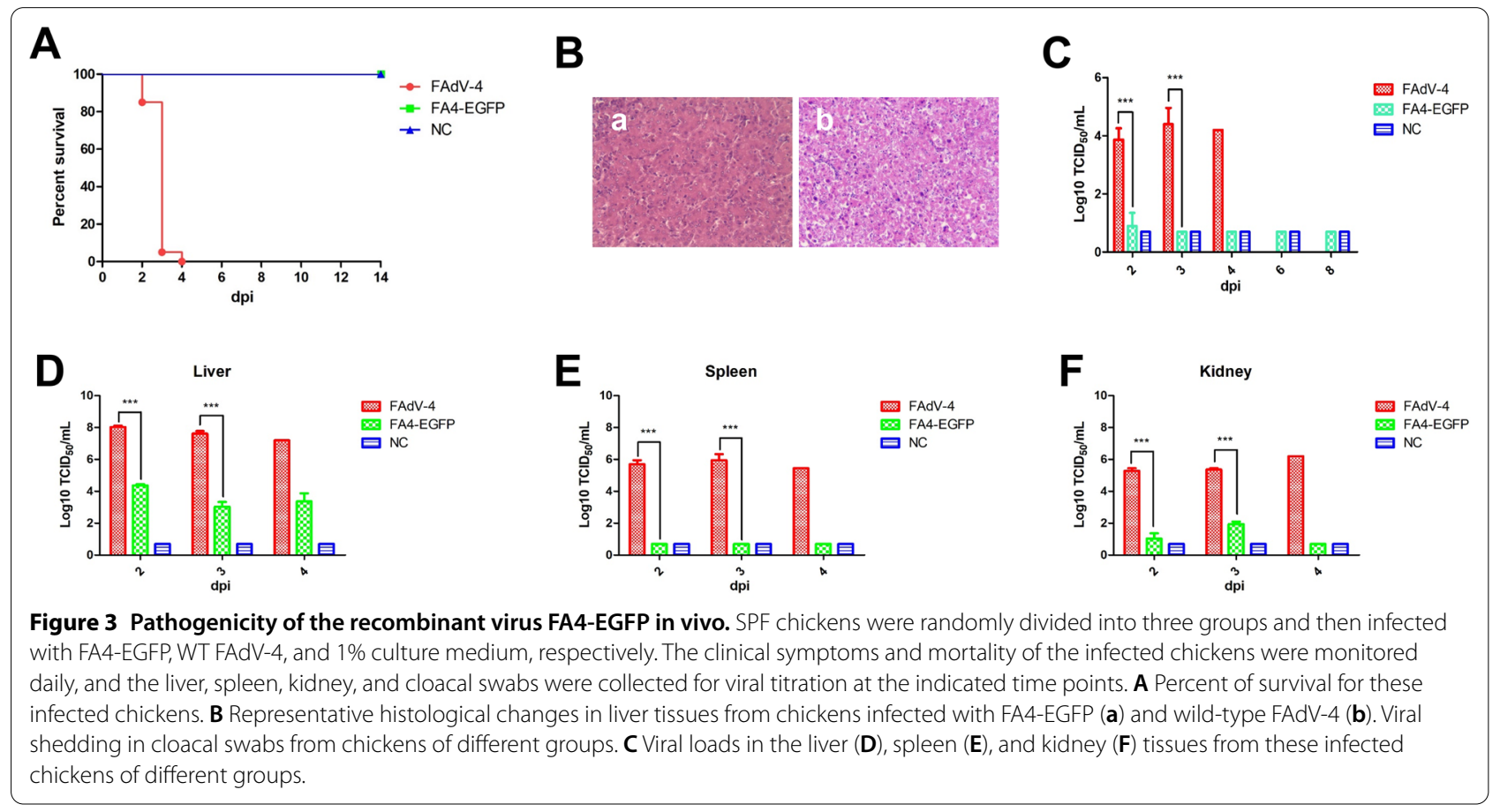


shed any viruses in cloaca at $3-8 \mathrm{dpi}$. For virus loading in tissues, as described in Figure 3D, the viral titers in the liver from the chickens infected with FAdV-4 reached $10^{7}-10^{8} \mathrm{TCID}_{50} / \mathrm{mL}$ at $2-4 \mathrm{dpi}$, whereas those in chickens infected with FA4-EGFP were only about $10^{2}-10^{4}$ $\mathrm{TCID}_{50} / \mathrm{mL}$.

Similarly, as shown in Figures 3E, F, a high viral titer of FAdV-4 was detected in the spleen and kidney, whereas a very low titer of FA4-EGFP was detected in the kidney, and no virus was detected in the spleen. These data from the virus cloacal shedding and tissue loading were consistent with the clinical signs and survival data. All these reveal that the FA4-EGFP virus is highly attenuated in chickens.

\section{FA4-EGFP provides efficient protection against lethal challenge of FAdV-4}

To evaluate the protective efficacy of the recombinant virus FA4-EGFP, the chickens previously inoculated with FA4-EGFP were challenged with a lethal dose of FAdV-4 at $21 \mathrm{dpi}$. The clinical signs and mortality of the challenged chickens were monitored daily, and the liver, spleen, kidney, and cloacal swabs were collected for viral titration at different time points post-challenge. After the challenge, the control group's chickens exhibited clinical signs, including depression, loss of appetite, and huddling together with ruffled feathers. These challenged control chickens' mortality reached $80 \%(21 / 26)$ at day 6 post-challenge (Figure 4A), whereas the challenged chickens previously inoculated with FA4-EGFP did not show any clinical symptoms, and all survived. Besides, necropsy analysis shows that hydropericardium and hepatitis were found in the challenged control group, but not in the challenged chickens previously inoculated with FA4-EGFP (Figure 4B). Moreover, high viral titers in cloacal swabs, the liver, spleen, and kidney from the challenged control group were detected at $2-4 \mathrm{dpc}$ (postchallenge), whereas no virus could be detected at all the time points tested in the challenged chickens previously inoculated with FA4-EGFP in cloacal swabs, liver, spleen, and kidney. All these results demonstrate that FA4-EGFP can be an efficient vaccine candidate against the highly pathogenic FAdV-4.

\section{FA4-EGFP induced more robust neutralizing activity than inactivated vaccine}

To further compare the protective efficacy of FA4-EGFP with the inactivated FAdV-4 vaccine candidate, 2-week old SPF chickens were inoculated with either one of the three doses of FA4-EGFP $\left(10^{6} \mathrm{TCID}_{50}, 10^{5} \mathrm{TCID}_{50}, 10^{4}\right.$ $\mathrm{TCID}_{50}$ ), or the inactivated FAdV-4 containing $5 \times 10^{6}$ $\mathrm{TCID}_{50}$ of viruses, respectively. The neutralizing activity (NT) of sera from the inoculated chickens were tested, and then these chickens were challenged with the lethal dose of FAdV-4 at 21 dpi. As described in Figure 5A, the average NT titer for sera at $7 \mathrm{dpi}$ was $1.5,1.8$, and 1.3 in

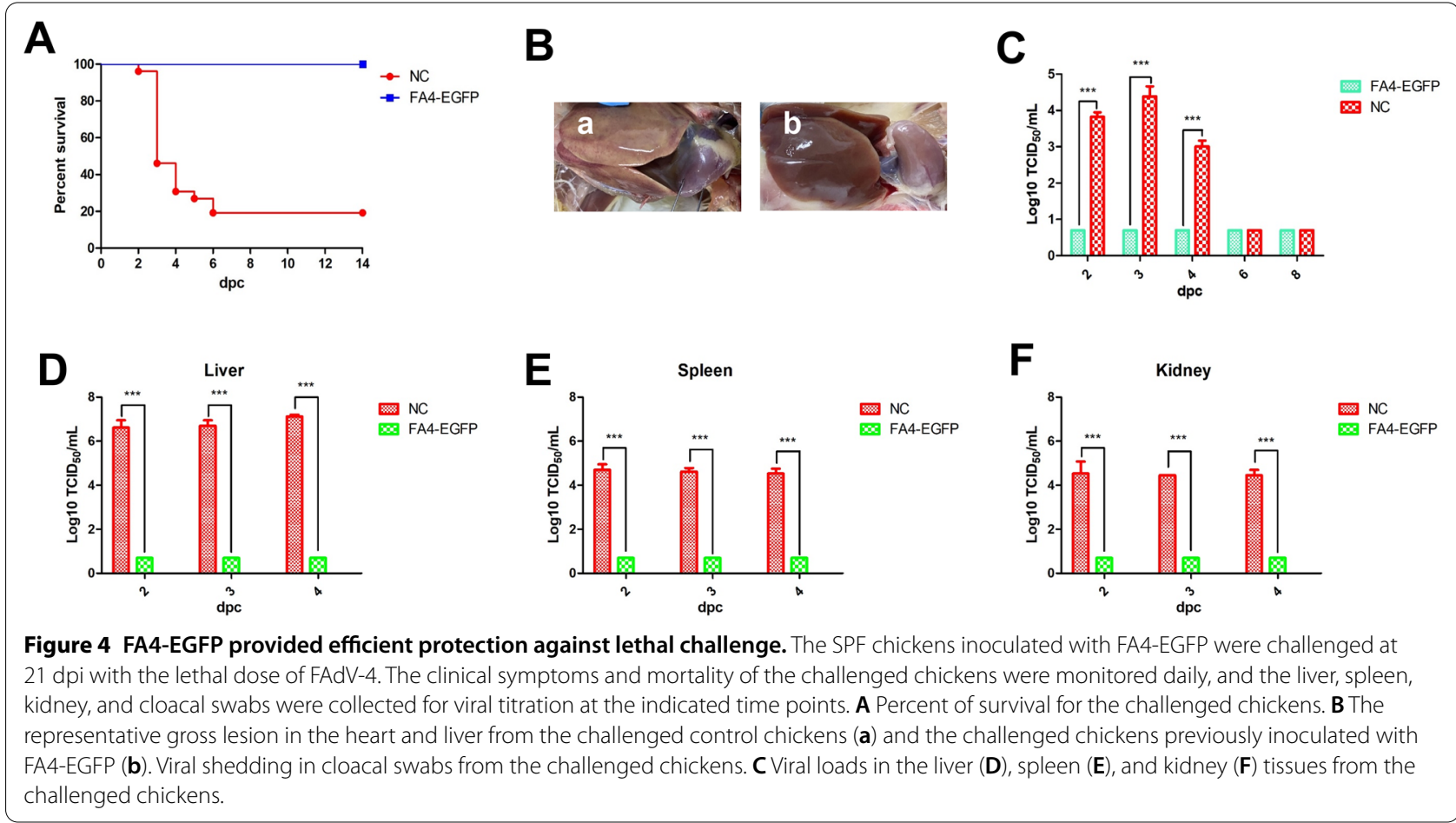




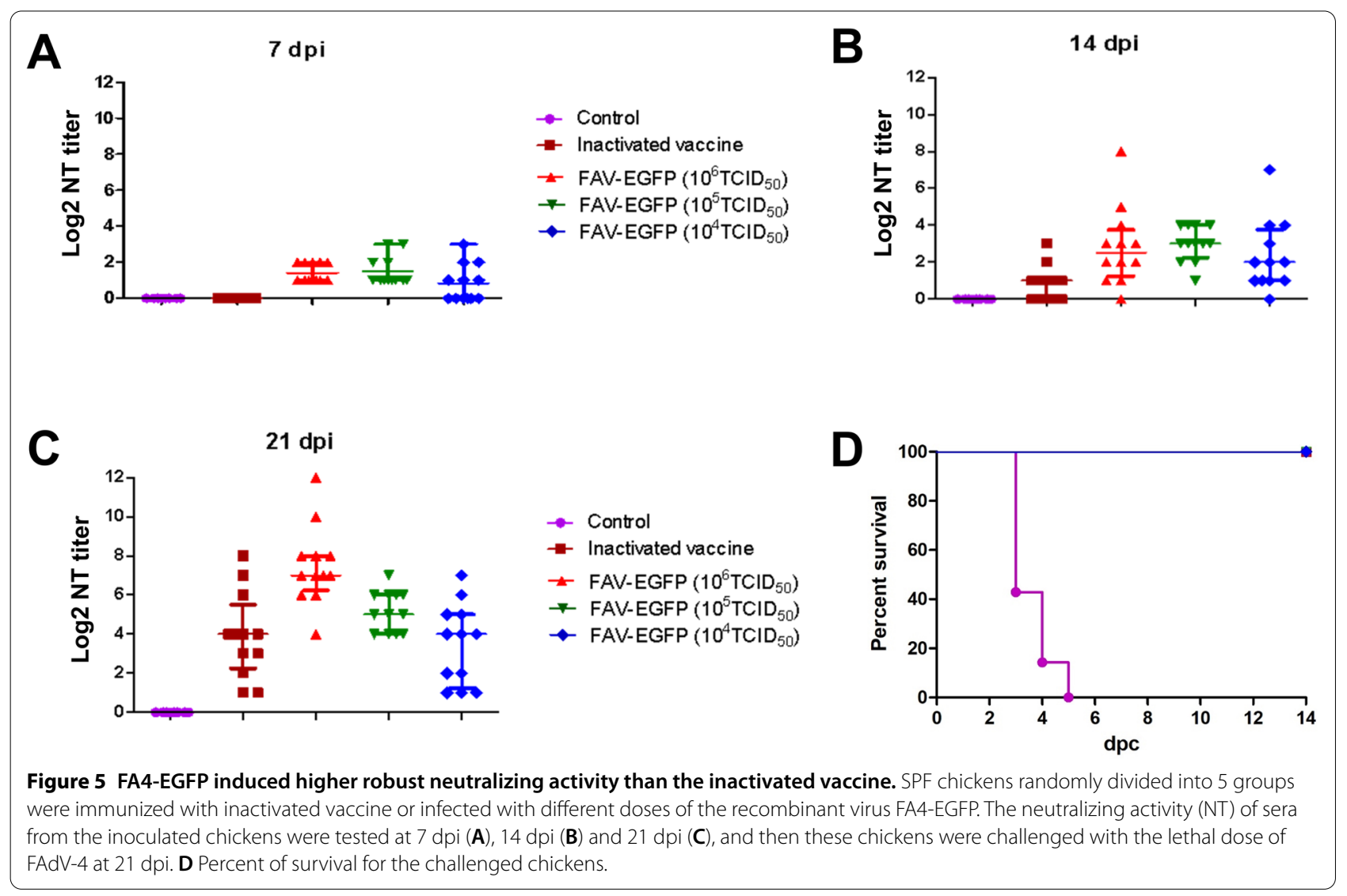

chickens inoculated with $10^{6} \mathrm{TCID}_{50}, 10^{5} \mathrm{TCID}_{50}$, and $10^{4} \mathrm{TCID}_{50}$ of FA4-EGFP respectively, whereas that in the control chickens and chickens inoculated with the inactivated vaccine could not be detected. The average NT titer for sera in chickens inoculated with $10^{6}$ $\mathrm{TCID}_{50}, 10^{5} \mathrm{TCID}_{50}$, and $10^{4} \mathrm{TCID}_{50}$ of FA4-EGFP was $2.8,3.0$, and 2.3 at $14 \mathrm{dpi}$, respectively, and $7.5,5.2$, and 3.5 at $21 \mathrm{dpi}$, respectively, whereas that in the chickens inoculated with the inactivated vaccine was 0.8 at $14 \mathrm{dpi}$ and 3.9 at 21 dpi as shown in Figures 5B, C. Moreover, inoculation with FA4-EGFP or the inactivated FAdV-4 provided efficient protection against lethal challenge at $21 \mathrm{dpi}$, whereas the challenged control chickens died at $5 \mathrm{dpc}$ as described in Figure 5D. These data demonstrate that except for efficient protection, FA4-EGFP induces neutralizing antibodies with higher titers earlier than the inactivated FAdV-4 vaccine candidate.

\section{Discussion}

The development of vaccines is one of the most efficient strategies to prevent and control diseases. Since the first outbreak of FAdV-4 in 1987 [18], various vaccine candidates, including inactivated [19-22], live-attenuated [11, 23-25], and recombinant subunit vaccines [26-30], have been generated. Different vaccines have unique advantages and disadvantages. Although the inactivated vaccine is highly safe and protective, the preparation of a high titer of the inactivated FAdV-4 is highly expensive. The cost for the generation of subunit vaccine is less expensive; however, the subunit vaccine's protective efficacy is generally limited compared with the whole inactivated FAdV-4 vaccines. Notably, both the inactivated vaccine and the sub-unit vaccine mainly induce a humoral immune response, but not a $\mathrm{T}$ cell immune response. Live-attenuated vaccines can trigger humoral and cellular immune responses to provide consistent and strong immune protection against viruses. Mansoor et al. [23] and Schonewille et al. [11] used chicken embryos and QT-35 cells to passage the pathogenic FAdV-4 to generate live-attenuated FAdV-4 vaccine candidates. Recently, Grgic et al. [24] used a non-pathogenic FAdV-4 isolate as a live-attenuated FAdV-4 vaccine candidate. Although these live-attenuated FAdV-4 vaccine candidates could provide efficient protection against the pathogenic FAdV-4, the molecular mechanism for the attenuation of these adapted strains or non-pathogenic strains need to be further elucidated. In this study, we targeted the fiber-2 gene, one of the virulent determiners 
for the pathogenesis of FAdV-4, to generate a recombinant virus FA4-EGFP carrying the EGFP-Fiber-2 fusion protein. Our in vitro and in vivo studies demonstrate that FA4-EGFP not only was highly attenuated but also could provide full protection against lethal challenge with the highly pathogenic FAdV-4.

The CRISPR/Cas 9 technique has been widely used to edit the host genes and modify the virus genes [31-34]. This study combined the homology-dependent knock-in and the CRISPR/Cas 9 approach to generate the recombinant virus FA4-EGFP. During the rescuing of the recombinant virus FA4-EGFP, the virus plagues with EGFP could be observed at $24 \mathrm{hpi}$ in the transfected LMH cells with the infection of FAdV-4, highlighting that the strategy used in this study to generate recombinant FAdV-4 was very efficient, and the $\mathrm{N}$-terminus of the fiber- 2 gene could be an efficient insertion site for expressing the foreign gene. Moreover, we found that the purified FA4EGFP passaged in LMH cells for more than 10 passages was very stable with EGFP-Fiber-2 fusion protein expression and without the reversion into the WT FAdV-4 (Data not shown). Also, we used PCR to detect the purity of FA-EGFP in the livers, kidneys and cloacal swabs of chickens infected the FA-EGFP. Only the specific band for FA-EGFP could be efficiently amplified whereas the band specific to the WT FAdV-4 could not be amplified in these samples (data not shown). All these demonstrate that the purity of FA-EGFP is robust. Notably, except for the large band of the EGFP-Fiber-2 protein, a small band was also detected in the LMH cells infected with the purified FAdV-4 by mAb against Fiber- 2 in Western blot (Figure 2B). The molecular weight of the small band detected in LMH cells infected with FA4-EGFP was very similar to the small band of Fiber-2 (two bands with similar molecular weights) in LMH cells infected with WT FAdV-4. A sequence assay further revealed that a potential truncated ORF initiated at the nucleotide 94 of fiber-2 was found, which has never been reported. The potential truncated ORF in fiber-2 might encode the small band of detected Fiber-2. Of course, whether fiber-2 can encode two proteins needs to be further elucidated.

Moreover, it should be noted that infection with a high dose $\left(10^{6} \mathrm{TCID}_{50}\right)$ of FA4-EGFP did not cause any clinical signs and all the infected chickens survived, whereas all the chickens infected with the same dose of WT FAdV-4 demonstrated severe clinical signs with HHS and died at $4 \mathrm{dpi}$, which highlights the significant attenuation of FA4-EGFP. In comparison with the inactivated FAdV-4 vaccine, even 50 or 500 times fewer doses of the live FA4-EGFP can induce earlier and stronger neutralizing antibody with efficient protection against the lethal challenge with FAdV-4, highlighting the potential application of the live-attenuated FA4-EGFP for controlling the disease caused by FAdV-4 could significantly save the cost for the generation of the high dose of the inactivated FAdV-4. Although the induced neutralizing antibody by the live attenuated FA4-EGFP in this study may contribute to an efficient protection, the molecular mechanism for the protection against FAdV-4 needs to be further investigated. Notably, Schonewille et al. reported that the QT-35 adapted live FAdV-4 vaccine candidate could provide efficient protection without inducing detectable neutralizing antibodies [11]. Schachner et al. also found the recombinant Fiber-2 subunit vaccine could not produce neutralizing antibodies but provide $96 \%$ protection against the lethal challenge of FAdV-4 [27]. Therefore, whether an efficient protection against FAdV-4 requires the induced neutralizing antibody needs to be further elucidated.

In summary, this is the first demonstration of the generation of the highly attenuated recombinant virus FA4EGFP expressing EGFP-Fiber-2 fusion protein and its efficient protective efficacy against lethal challenge with FAdV-4. Compared with the inactivated FAdV-4 vaccine, FA4-EGFP could induce neutralizing antibodies with higher titers earlier. However, the molecular basis for the attenuation of FA4-EGFP needs to be further elucidated. Does the fusion protein of EGFP-Fiber-2 expressed in FA4-EGFP affect the interaction between Fiber- 2 and Penton or other host proteins? Can other foreign antigens replace EGFP to generate dual vaccines against both FAdV-4 and other pathogens? Additionally, except for inducing an efficient humoral immune response with high neutralizing antibodies, the protective efficacy for cellular immunity against FAdV-4 for FA4-EGFP also needs to be further investigated.

\section{Supplementary Information}

The online version contains supplementary material available at https://doi. org/10.1186/s13567-021-00907-z.

Additional file 1. Complete sequence of EGFP_fiber-2.

\section{Abbreviations}

FAdV-4: Fowl adenovirus serotype 4; HHS: Hydropericardium-hepatitis syndrome; IBH: Inclusion body hepatitis; GEU: Gizzard erosion and ulceration; LMH: Leghorn male hepatoma; mAb: Monoclonal antibody; HA: Homologous arm; hpi: Hour post-infection; dpi: Day post-infection; dpc: Day post-challenge; NC: Nitrocellulose; RT: Room temperature; DMEM: Dulbecco modified Eagle medium; FBS: Fetal bovine serum; IFA: Indirect immunofluorescent assay.

\section{Acknowledgements}

We thank Jianjun Zhang (Sinopharm Yangzhou VAC Biological Engineering Co.Ltd) for kindly providing us SPF chickens.

\section{Authors' contributions}

$\mathrm{QX}, J Y$, and AQ designed the project; QX, SC, WZ, WW, LL, QK, HF, and TL carried out the experiments; QX, SC, JY, ZW, WG, and HS analyzed the data; QX, JY, and TG drafted the manuscript; JY supervised all the experiments and participated 
in the data analysis; QX, ZW, WG, HS, and AQ discussed and prepared the final report. All authors read and approved the final manuscript.

\section{Funding}

This study was supported by Jiangsu Agricultural Science and Technology Innovation Fund (CX(19)3026), the Key Research \& Development (R\&D) Plan in Yangzhou City (YZ2020052), Science and Innovation Program for college students (201911117009Z), Key Laboratory of Prevention and Control of Biological Hazard Factors (Animal Origin) for Agrifood Safety and Quality (26116120), Research Foundation for Talented Scholars in Yangzhou University and the Priority Academic Program Development of Jiangsu Higher Education Institutions.

\section{Availability of data and materials}

The datasets used and/or analyzed during the current study are available from the corresponding author on reasonable request.

\section{Ethics approval and consent to participate}

All animal experiments complied with the institutional animal care guidelines and the protocol (SYXY-19), which was approved by the Animal Care Committee of Yangzhou University. At the end of the experiment, all the chickens were euthanized by $\mathrm{CO}_{2}$.

\section{Competing interests}

The authors declare that they have no competing interests.

\section{Author details}

${ }^{1}$ Key Laboratory of Jiangsu Preventive Veterinary Medicine, Key Laboratory for Avian Preventive Medicine, Ministry of Education, College of Veterinary Medicine, Yangzhou University, Yangzhou 225009, Jiangsu, China. ${ }^{2}$ Jiangsu Co-Innovation Center for Prevention and Control of Important Animal Infectious Diseases and Zoonoses, Yangzhou 225009, Jiangsu, China. ${ }^{3}$ Joint International Research Laboratory of Agriculture and Agri-Product Safety, the Ministry of Education of China, Yangzhou University, Yangzhou 225009, Jiangsu, China. ${ }^{4}$ Institute of Agricultural Science and Technology Development, Yangzhou University, Yangzhou 225009, Jiangsu, China. ${ }^{5}$ Sinopharm Yangzhou VAC Biological Engineering Co.Ltd, Yangzhou 225127, Jiangsu, China. ${ }^{6}$ College of Animal Science and Technology, Yangzhou University, Yangzhou 225009, Jiangsu, China.

Received: 17 November 2020 Accepted: 21 January 2021

Published online: 27 February 2021

\section{References}

1. Hess M (2020) Aviadenovirus infections. In: Swayne DE, Boulianne M, Logue CM, McDougald LR, Nair V, Suarez DL (eds) Diseases of poultry, $14^{\text {th }}$ edn. Wiley-Blackwell, Hoboken

2. Hess M (2000) Detection and differentiation of avian adenoviruses: a review. Avian Pathol 29:195-206

3. Schachner A, Matos M, Grafl B, Hess M (2018) Fowl adenovirus-induced diseases and strategies for their control-a review on the current global situation. Avian Pathol 47:111-126

4. Mazaheri A, Prusas C, Voss M, Hess M (1998) Some strains of serotype 4 fowl adenoviruses cause inclusion body hepatitis and hydropericardium syndrome in chickens. Avian Pathol 27:269-276

5. Zhang HW, Jin WJ, Ding K, Cheng XC, Sun YR, Wang JK, Cheng SP, Wu H, Zhang CJ (2017) Genetic characterization of fowl adenovirus strains isolated from poultry in China. Avian Dis 61:341-346

6. Cui J, Xu YY, Zhou ZT, Xu QR, Wang JX, Xiao YC, Li ZL, Bi DR (2020) Pathogenicity and molecular typing of fowl adenovirus-associated with hepatitis/hydropericardium syndrome in central China (2015-2018). Front Vet Sci 7:190

7. Ye J, Liang G, Zhang J, Wang W, Song N, Wang P, Zheng W, Xie Q, Shao H, Wan Z, Wang C, Chen H, Gao W, Qin A (2016) Outbreaks of serotype 4 fowl adenovirus with novel genotype China. Emerg Microbes Infect 5:e50

8. Li PH, Zheng PP, Zhang TF, Wen GY, Shao HB, Luo QP (2017) Fowl adenovirus serotype 4: epidemiology, pathogenesis, diagnostic detection, and vaccine strategies. Poult Sci 96:2630-2640
9. Shah MS, Ashraf A, Khan MI, Rahman M, Habib M, Chughtai MI, Qureshi JA (2017) Fowl adenovirus: history, emergence, biology and development of a vaccine against hydropericardium syndrome. Arch Virol 162:1833-1843

10. Zhao J, Zhong Q, Zhao Y, Hu YX, Zhang GZ (2015) Pathogenicity and complete genome characterization of fowl adenoviruses isolated from chickens associated with inclusion body hepatitis and hydropericardium syndrome in China. PLoS One 10:e0133073

11. Schonewille E, Jaspers R, Paul G, Hess M (2010) Specific-pathogen-free chickens vaccinated with a live FAdV-4 vaccine are fully protected against a severe challenge even in the absence of neutralizing antibodies. Avian Dis 54:905-910

12. Manzoor S, Hussain Z, Rahman SU, Hussain I (2013) Identification of antibodies against hydropericardium syndrome in wild birds. Br Poult Sci 54:325-328

13. Marek A, Nolte V, Schachner A, Berger E, Schlotterer C, Hess M (2012) Two fiber genes of nearly equal lengths are a common and distinctive feature of Fowl adenovirus C members. Vet Microbiol 156:411-417

14. Wang W, Liu Q, Li T, Geng T, Chen H, Xie Q, Shao H, Wan Z, Qin A, Ye J (2020) Fiber-1, not fiber-2, directly mediates the infection of the pathogenic serotype 4 fowl adenovirus via its shaft and knob domains. J Virol 94:e00954-e1020

15. Pan Q, Wang J, Gao YL, Wang Q, Cui HY, Liu CJ, Qi XL, Zhang YP, Wang YQ, Li K, Gao L, Liu AJ, Wang XM (2020) Identification of chicken CAR homology as a cellular receptor for the emerging highly pathogenic fowl adenovirus 4 via unique binding mechanism. Emerg Microbes Infect 9:586-596

16. Zhang Y, Liu R, Tian K, Wang Z, Yang X, Gao D, Zhang Y, Fu J, Wang H, Zhao $J(2018)$ Fiber2 and hexon genes are closely associated with the virulence of the emerging and highly pathogenic fowl adenovirus 4. Emerg Microbes Infect 7:199

17. Ran FA, Hsu PD, Wright J, Agarwala V, Scott DA, Zhang F (2013) Genome engineering using the CRISPR-Cas9 system. Nat Protoc 8:2281-2308

18. Anjum AD, Sabri MA, labal Z (1989) Hydropericarditis syndrome in broiler chickens in Pakistan. Vet Rec 124:247-248

19. Chishti MA, Afzal M, Cheema AH (1989) Preliminary studies on the development of vaccine against the "hydropericardium syndrome" of poultry. Rev Sci Tech 8:797-801

20. Afzal M, Ahmad I (1990) Efficacy of an inactivated vaccine against hydropericardium syndrome in broilers. Vet Rec 126:59-60

21. Anjum AD (1990) Experimental transmission of hydropericardium syndrome and protection against it in commercial broiler chickens. Avian Pathol 19:655-660

22. Kim MS, Lim TH, Lee DH, Youn HN, Yuk SS, Kim BY, Choi SW, Jung CH, Han $\mathrm{JH}$, Song CS (2014) An inactivated oil-emulsion fowl Adenovirus serotype 4 vaccine provides broad cross-protection against various serotypes of fowl Adenovirus. Vaccine 32:3564-3568

23. Mansoor MK, Hussain I, Arshad M, Muhammad G (2011) Preparation and evaluation of chicken embryo-adapted fowl adenovirus serotype 4 vaccine in broiler chickens. Trop Anim Health Prod 43:331-338

24. Grgic H, Poljak Z, Sharif S, Nagy E (2013) Pathogenicity and cytokine gene expression pattern of a serotype 4 fowl adenovirus isolate. PLoS One 8:e77601

25. Schonewille E, Singh A, Gobel TW, Gerner W, Saalmuller A, Hess M (2008) Fowl adenovirus (FAdV) serotype 4 causes depletion of $B$ and $T$ cells in lymphoid organs in specific pathogen-free chickens following experimental infection. Vet Immunol Immunopathol 121:130-139

26. Shah MS, Ashraf A, Rahman M, Khan MI, Qureshi JA (2012) A subunit vaccine against hydropericardium syndrome using adenovirus penton capsid protein. Vaccine 30:7153-7156

27. Schachner A, Marek A, Jaskulska B, Bilic I, Hess M (2014) Recombinant FAdV-4 fiber-2 protein protects chickens against hepatitis-hydropericardium syndrome (HHS). Vaccine 32:1086-1092

28. Shah MS, Ashraf A, Khan MI, Rahman M, Habib M, Qureshi JA (2016) Molecular cloning, expression and characterization of 100K gene of fowl adenovirus-4 for prevention and control of hydropericardium syndrome. Biologicals 44:19-23

29. Chen L, Yin L, Zhou Q, Li Q, Luo Y, Xu Z, Zhang Y, Xue C, Cao Y (2018) Immunogenicity and protective efficacy of recombinant fiber-2 protein in protecting SPF chickens against fowl adenovirus 4. Vaccine 36:1203-1208 
30. Wang X, Tang Q, Chu Z, Wang P, Luo C, Zhang Y, Fang X, Qiu L, Dang R, Yang Z (2018) Immune protection efficacy of FAdV-4 surface proteins fiber-1, fiber-2, hexon and penton base. Virus Res 245:1-6

31. Bi YW, Sun L, Gao DD, Ding C, Li ZH, Li YD, Cun W, Li QH (2014) High-efficiency targeted editing of large viral genomes by RNA-guided nucleases. PLoS Pathog 10:e1004090

32. Bierle CJ, Anderholm KM, Ben Wang J, McVoy MA, Schleiss MR (2016) Targeted mutagenesis of Guinea pig cytomegalovirus using CRISPR/ Cas9-mediated gene editing. J Virol 90:6989-6998

33. Yuen KS, Wang ZM, Wong NHM, Zhang ZQ, Cheng TF, Lui WY, Chan CP, Jin DY (2018) Suppression of Epstein-Barr virus DNA load in latently infected nasopharyngeal carcinoma cells by CRISPR/Cas9. Virus Res 244:296-303
34. Luo J, Teng M, Zai XS, Tang N, Zhang YY, Mandviwala A, Reddy VRAP, Baigent S, Yao YX, Nair V (2020) Efficient mutagenesis of Marek's disease virus-encoded microRNAs using a CRISPR/Cas9-based gene editing system. Viruses 12:466

\section{Publisher's Note}

Springer Nature remains neutral with regard to jurisdictional claims in published maps and institutional affiliations.
Ready to submit your research? Choose BMC and benefit from:

- fast, convenient online submission

- thorough peer review by experienced researchers in your field

- rapid publication on acceptance

- support for research data, including large and complex data types

- gold Open Access which fosters wider collaboration and increased citations

- maximum visibility for your research: over 100M website views per year

At BMC, research is always in progress.

Learn more biomedcentral.com/submissions 